\title{
Poorly Differentiated Thyroid Carcinoma Patients with Detectable Thyroglobulin Levels after Initial Treatment Show an Increase in Mortality and Disease Recurrence
}

\author{
Tiago Nunes da Silva Edward Limbert Valeriano Leite \\ Instituto Português de Oncologia de Lisboa Francisco Gentil, Lisbon, Portugal
}

\section{Keywords \\ Poorly differentiated thyroid carcinoma - Thyroglobulin level $\cdot$ Survival rate $\cdot$ Disease recurrence}

\begin{abstract}
Purpose: The role of thyroglobulin $(\mathrm{Tg})$ in predicting death and recurrence risk in patients with poorly differentiated thyroid carcinoma (PDTC) is not well established. We aimed to analyze Tg levels following total thyroidectomy and adjuvant radioiodine treatment (RAI) in PDTC patients and correlate $\mathrm{Tg}$ levels with survival and recurrence. Methods: A retrospective analysis was conducted on 101 patients with PDTC who were treated between 1986 and 2010. Among them, 38 had no distant metastases at presentation, were managed by total thyroidectomy and adjuvant RAl, and had negative anti-Tg antibodies. An unstimulated Tg level $<1 \mathrm{ng} /$ $\mathrm{mL}$ was used as a cut-off point for undetectable Tg levels. Association of patient and tumor characteristics with Tg levels was examined by $x^{2}$ test. Overall survival, disease-specific survival (DSS), and recurrence-free survival (RFS), stratified by $\mathrm{Tg}$ levels, were calculated by the Kaplan-Meier method and compared by the log-rank test. Results: Compared to patients with undetectable $\mathrm{Tg}$, cases with detectable $\mathrm{Tg}$ had
\end{abstract}

\section{KARGER}

E-Mail karger@karger.com

www.karger.com/etj a lower probability of achieving free surgical margins (21.7 vs. $46.7 \% ; p=0.04)$, higher node status ( 73.3 vs. $21.8 \% ; p=$ 0.005 ), decreased 5-year DSS (65 vs. $100 \% ; p=0.009$ ), and worse 5 -year RFS (32 vs. $84 \%, p=0.010$ ), with a significant number of patients having a recurrence in the first year (50 vs. $12.5 \% ; p=0.021$ ). Patients with detectable Tg levels also showed worse locoregional (55.6 vs. $90.9 \% ; p=0.014$ ) and distant control (5-year distant control of 46.9 vs. $91 \%$; $p=$ 0.017). Conclusions: Our results suggest that detectable $\mathrm{Tg}$ levels after surgery and RAI in a subset of PDTC patients appear to predict a higher rate of death and recurrence.

(c) 2018 European Thyroid Association Published by S. Karger AG, Basel

\section{Introduction}

Poorly differentiated thyroid carcinoma (PDTC) is a rare type of thyroid cancer accounting for $<6 \%$ of all thyroid malignancies [1]. Nevertheless, it is the second cause of death from follicular cell-derived thyroid cancer after anaplastic carcinoma [2]. Death is usually secondary to metastatic disease [3]. The tumors show significant clinical, biological, and pathological heterogeneity, with char- 
acteristics in between differentiated thyroid carcinoma (DTC) and anaplastic carcinoma [4, 5]. Despite being less differentiated than DTCs, these tumors partially retain the ability to produce colloid and thyroglobulin (Tg) [68]. The prognostic role of Tg in PDTC was unknown due to the very limited number of available studies until the work of Ibrahimpasic et al. [9], who showed that patients with detectable Tg levels had higher a rate of disease recurrence than those with undetectable Tg but found no effect on mortality. The objective of this study was to correlate Tg values in PDTC patients following total thyroidectomy and adjuvant radioiodine treatment (RAI) with patient characteristics, tumor aggressiveness, disease recurrence, and mortality.

\section{Study Design}

We performed a retrospective study at the Portuguese Institute of Oncology Francisco Gentil, Lisbon Centre (IPOLFG), the largest referral center for thyroid cancer in Portugal, with the aim of evaluating the predictive role of Tg levels on recurrence and survival following total thyroidectomy and adjuvant RAI in PDTC patients. The study was conducted in accordance with the principles of the Declaration of Helsinki and approved by the Institutional Review Board of our institution. Informed consent for management of clinical data was obtained from all individual patients included in the study.

\section{Patients}

A total of 101 PDTC patients were diagnosed and treated at IPOLFG between January 1986 and December 2010. Thirty-eight patients fulfilled the following inclusion criteria: no distant metastases at presentation (M0), treatment by total thyroidectomy and RAI, and available Tg levels with negative anti-Tg antibodies measured between 3 and 9 months after adjuvant RAI. No adjuvant radiotherapy or systemic therapy was used in these cases.

\section{Protocol}

Medical records were reviewed for patient and tumor characteristics, including the year of diagnosis, gender, age at diagnosis, tumor pathology, TNM staging, time of follow-up, time of first recurrence, and the duration of survival from the date of diagnosis. Recurrence was defined as a new diagnosis of locoregional or distant tumor by tissue biopsy or imaging at least 6 months after initial therapy.
Data regarding disease-specific mortality were reviewed based on the hospital records or death certificates. Tumor size was defined as the maximal diameter of the surgical specimen, or, if this was not available, that from the previous imaging studies. The criteria in the AJCCC Cancer Staging Manual (ed 7) were used for staging [10]. Neck dissection was performed in selected patients for suspected lymph node disease at the time of surgery. The Turin proposal criteria were used to define the PDTC diagnosis as previously described [11]. An unstimulated Tg level $<1 \mathrm{ng} /$ $\mathrm{mL}$ after surgery and RAI was used as a cut-off point for undetectable Tg levels. This cut-off point was chosen to encompass the known functional sensitivities of the different assays used in our institution over time, i.e., SELco Tg Medipan Diagnostica (a functional sensitivity of 0.5-1 ng/ $\mathrm{mL}$ ) and Immulite 2000-Thyroglobulin DCP/Siemens (a functional sensitivity of $0.9 \mathrm{ng} / \mathrm{mL}$ ).

\section{Statistical Analysis}

Statistical analyses were performed using IBM SPSS Statistics for Windows v21.0 (IBM Corp., Armonk, NY, USA). The $\chi^{2}$ test was used to compare the differences in patient and tumor characteristics between those with detectable and undetectable Tg levels.

Five-year overall survival (OS), disease-specific survival (DSS), and recurrence-free survival (RFS) were calculated by the Kaplan-Meier method and compared by log-rank test between those with a detectable and undetectable Tg value. Statistical significance was defined as $p<0.05$.

\section{Results}

\section{RAI and Histological Phenotype in Patients with}

Detectable and Undetectable $\mathrm{Tg}$

The study included 38 cases from 1986 to 2011 (patient 25 in June 1997, median in January 2002, and patient 75 in October 2008).

After total thyroidectomy and RAI, 15 patients had detectable $(\mathrm{Tg}>1 \mathrm{ng} / \mathrm{mL}$ ) and 23 had undetectable ( $\mathrm{Tg} \leq 1$ $\mathrm{ng} / \mathrm{mL}$ ) Tg levels. Patients received a median cumulative activity of 8.362 GBq (range 1.85-20.757 GBq). Patients with detectable $\mathrm{Tg}$ and undetectable $\mathrm{Tg}$ levels received a median cumulative activity of RAI of $8.362 \mathrm{GBq}$ and $6.771 \mathrm{GBq}$, respectively ( $p=\mathrm{ns}$ ). Only 1 patient (with a pT1 tumor with R0 resection and detectable Tg after initial treatment) received an activity $<3.7 \mathrm{GBq}$.

The pathological subtypes in the 15 patients with detectable Tg were insular $(n=6)$, solid $(n=1)$, trabecular 
$(n=1)$, and mixed phenotype $(n=7)$. In the 23 patients with undetectable $\mathrm{Tg}$, these were insular $(n=10)$, solid $(n=3)$, trabecular $(n=3)$, and mixed phenotype $(n=7)$.

\section{Characteristics of PDTC Cases Stratified by Tg Group}

Patients with undetectable and detectable Tg levels showed no significant age or gender differences (Table 1). Around two-thirds of all cases were older than 45 years and the female-to-male ratio was also similar in both groups (Table 1).

Pathological tumor characteristics in the 2 groups were only statistically different for surgical margins and nodal status; patients with detectable $\mathrm{Tg}$ had a lower probability of achieving R0 margins ( 21.7 vs. $46.7 \%$; $p=$ 0.04 ) and a higher node status ( 73.3 vs. $21.8 \% ; p=0.005$ ). Nevertheless, these patients showed a trend towards more advanced local disease with a higher pT4 status (61 vs. $37.9 \% ; p=0.083)$ and more frequent extrathyroid extension ( 80 vs. $52.2 \%$; $p=0.082$ ). No statistical significant differences between the 2 groups were found regarding the size of primary tumor, vascular invasion, or histological subtype.

\section{Outcome of PDTC Patients Stratified by Tg Group}

In our cohort of 38 PDTC patients, 14 died (37\%), with 9 deaths (64\%) being specifically related to the disease (Table 1). The number of disease-related deaths was significantly higher in the group with detectable $\mathrm{Tg}$ (50 vs. $8.3 \% ; p=0.004)$. In the first year of follow-up, deaths were observed in $13 \%$ of the patients in the group with detectable Tg levels ( 2 cases) compared with no deaths in the group with undetectable $\mathrm{Tg}$ (the first death occurred after 8.7 years of follow-up).

There were 14 cases of recurrence (36\%): 7 locoregional and 7 metastatic. Patients with detectable Tg levels had a significant increase in recurrent disease (71.4 vs. 16.7\%; $p=0.001$ ). Both locoregional and distant recurrence were significantly increased in the group with detectable $\mathrm{Tg}$ levels ( 42.9 vs. $8.3 \% ; p=0.012$ and 35.7 vs. $8.3 \% ; p=0.036$, respectively). In the first year of follow-up, recurrence was observed in $50 \%$ of the group with detectable Tg levels and in $12.5 \%$ of the group with undetectable Tg levels ( 7 vs. 2 cases; $p=0.021$ ). These 2 patients with early recurrence despite negative Tg levels both had a status of pT4, with the largest tumor diameters of 52 and $60 \mathrm{~mm}$ with tracheal invasion in one case and positive Nla status in the other.

After a median follow-up of 7.35 years (range $0.71-$ 22.7 years), a trend towards a lower 5-year OS was observed in patients with detectable $\mathrm{Tg}$ (65 vs. $91.3 \%$; 6.9
Table 1. Characteristics stratified by Tg group

\begin{tabular}{|c|c|c|c|}
\hline & $\begin{array}{l}\text { Tg undetectable } \\
(n=23)\end{array}$ & $\begin{array}{l}\text { Tg detectable } \\
(n=15)\end{array}$ & $p$ value $^{\mathrm{a}}$ \\
\hline \multicolumn{4}{|l|}{ Age } \\
\hline$<45$ years & $33.3(8)$ & $33.3(5)$ & \multirow{2}{*}{0.633} \\
\hline$>45$ years & $65.2(15)$ & $66.7(10)$ & \\
\hline \multicolumn{4}{|l|}{ Sex } \\
\hline Female & $60.9(14)$ & $66.7(10)$ & \multirow[t]{2}{*}{0.792} \\
\hline Male & $39.1(9)$ & $33.3(5)$ & \\
\hline \multicolumn{4}{|c|}{ Tumor diameter } \\
\hline$\leq 4 \mathrm{~cm}$ & $50(12)$ & $40(6)$ & \multirow[t]{2}{*}{0.742} \\
\hline$>4 \mathrm{~cm}$ & $47.8(11)$ & $60(9)$ & \\
\hline \multicolumn{4}{|l|}{ pT stage } \\
\hline $1-3$ & $62.1(14)$ & $38.9(6)$ & \multirow[t]{2}{*}{0.083} \\
\hline 4 & $37.9(9)$ & $61.1(9)$ & \\
\hline \multicolumn{4}{|c|}{ Extra thyroid extension } \\
\hline Negative & $47.8(11)$ & $20(3)$ & \multirow[t]{2}{*}{0.082} \\
\hline Positive & $52.2(12)$ & $80(12)$ & \\
\hline \multicolumn{4}{|c|}{ Surgical margins } \\
\hline Negative & $65.2(15)$ & $33.3(5)$ & \multirow[t]{3}{*}{0.04} \\
\hline Positive & $21.7(5)$ & $46.7(7)$ & \\
\hline Unknown & $13(3)$ & $20(3)$ & \\
\hline \multicolumn{4}{|c|}{ Polymorphic histology } \\
\hline Negative & $88.9(16)$ & $81.8(9)$ & \multirow[t]{2}{*}{0.642} \\
\hline Positive & $11.1(2)$ & $18.2(2)$ & \\
\hline \multicolumn{4}{|c|}{ Vascular invasion } \\
\hline Negative & $21.8(5)$ & $13.4(2)$ & \multirow[t]{2}{*}{0.804} \\
\hline Positive & $78.2(18)$ & $86.6(13)$ & \\
\hline \multicolumn{4}{|c|}{ Polymorphic histology } \\
\hline Negative & $69.6(16)$ & $53.3(8)$ & \multirow[t]{2}{*}{0.642} \\
\hline Positive & $30.4(7)$ & $46.7(7)$ & \\
\hline \multicolumn{4}{|l|}{$\mathrm{pN}$ stage } \\
\hline 0 & $78.2(18)$ & $26.7(4)$ & \multirow[t]{2}{*}{0.005} \\
\hline 1 & $21.8(5)$ & $73.3(11)$ & \\
\hline \multicolumn{4}{|l|}{ Overall deaths } \\
\hline Yes & $25(6)$ & $57.1(8)$ & \multirow[t]{2}{*}{0.048} \\
\hline No & $75(18)$ & $42.9(6)$ & \\
\hline \multicolumn{4}{|c|}{ Disease-related deaths } \\
\hline Yes & $8.3(2)$ & $50(7)$ & \multirow[t]{2}{*}{0.004} \\
\hline No & $91.7(22)$ & $50(7)$ & \\
\hline Overall recurr & & & \\
\hline Yes & $16.7(4)$ & $71.4(10)^{\mathrm{b}}$ & 0.001 \\
\hline No & $83.3(20)$ & $28.6(4)$ & \\
\hline Local recurren & & & \\
\hline Yes & $8.3(2)$ & $42.9(6)$ & 0.012 \\
\hline No & $91.7(22)$ & $57.1(8)$ & \\
\hline Distant recurr & & & \\
\hline Yes & $8.3(2)$ & $35.7(5)$ & 0.036 \\
\hline No & $91.7(22)$ & $64.3(9)$ & \\
\hline
\end{tabular}

Values are expressed as \% (n). Tg, thyroglobulin.

${ }^{a}$ By means of the $\chi^{2}$ test.

${ }^{\mathrm{b}} \mathrm{A}$ patient in the detectable $\mathrm{Tg}$ group was found (at the same visit) to have both nodal and distant metastasis. As such, the sum of total recurrence is smaller than the sum of local and distant recurrence.
Increased Mortality and Recurrence in PDTC with Detectable Tg Levels
Eur Thyroid J 2018;7:313-318 DOI: $10.1159 / 000491996$ 


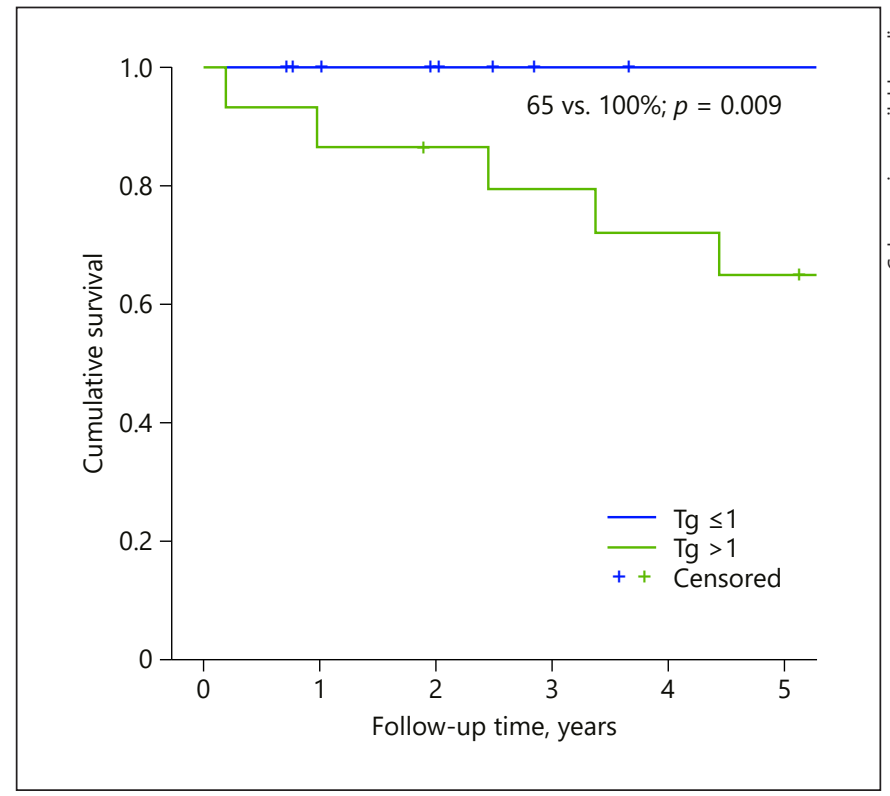

Fig. 1. Five-year disease-specific survival (DSS), stratified by Tg level.

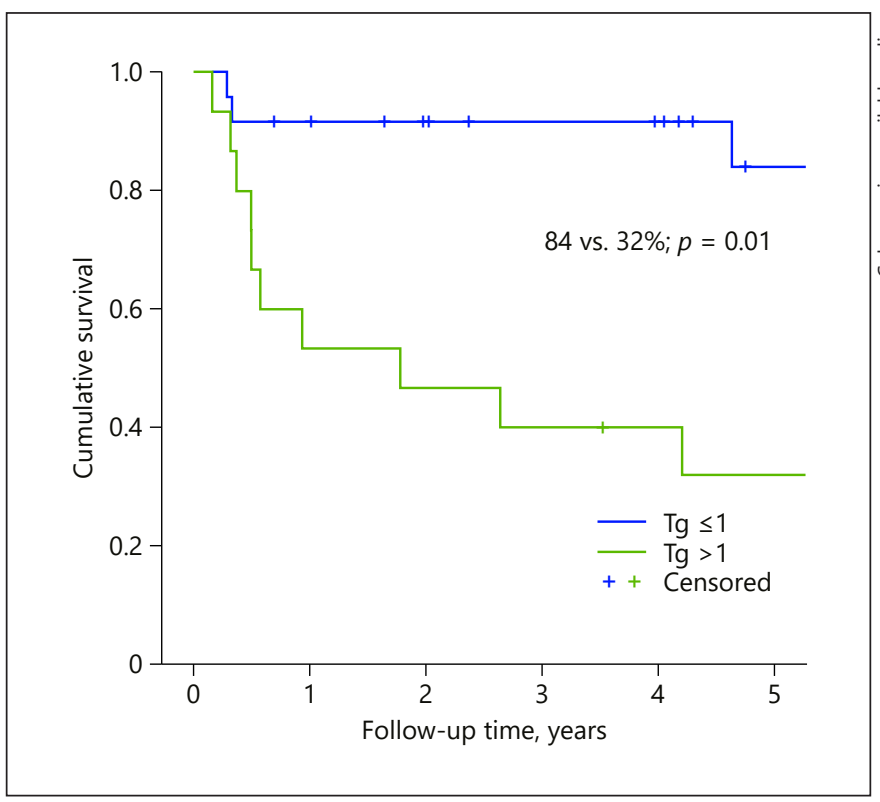

Fig. 2. Five-year recurrence-free survival (RFS), stratified by $\mathrm{Tg}$ level.

years vs. median not reached; $p=0.096$ ). As shown in Figure 1, patients with detectable Tg had a significant decrease in 5-year DSS (65 vs. 100\%; median 11.2 years vs. median not reached; $p=0.009$; Fig. 1 ), a significant decrease in 5-year RFS (32 vs. 84\%; median 1.7 years vs. me-

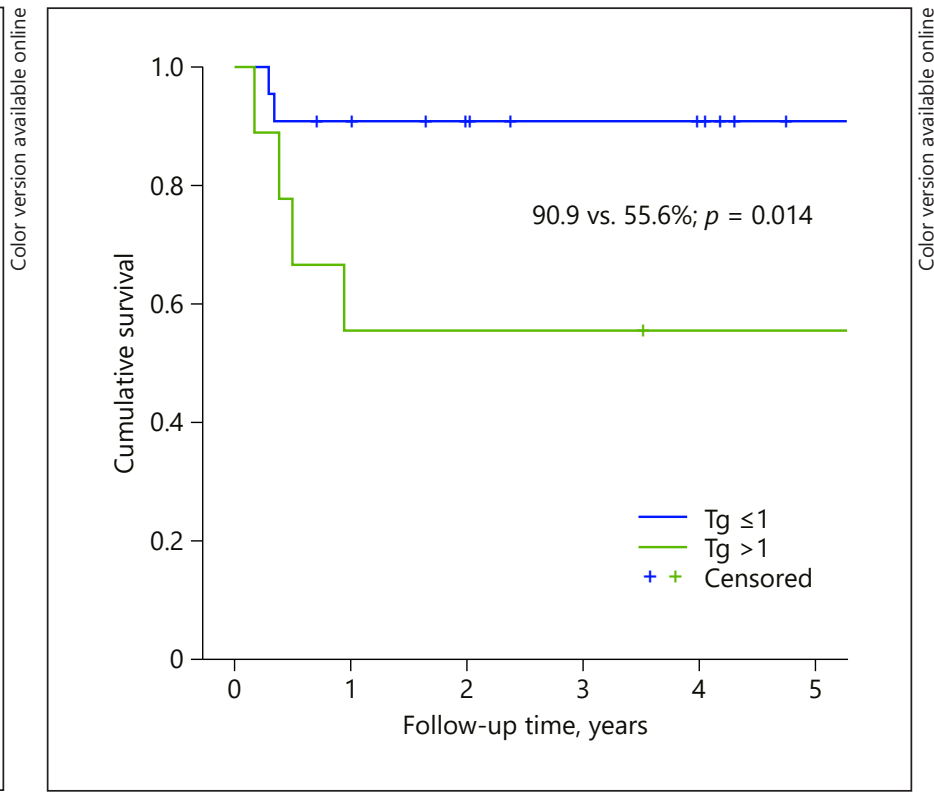

Fig. 3. Five-year regional recurrence-free survival (RFS), stratified by $\mathrm{Tg}$ level.

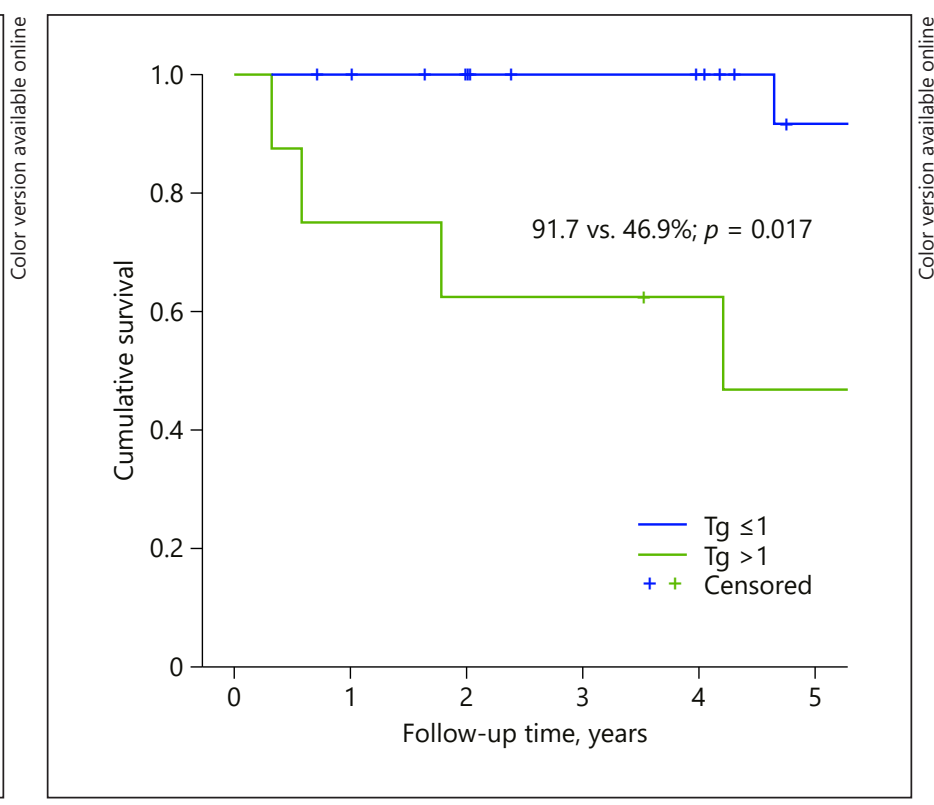

Fig. 4. Five-year distant recurrence-free survival (RFS), stratified by Tg level.

dian not reached; $p=0.01$; Fig. 2), a significant decrease in 5-year regional control (55.6 vs. 90.9\%; median 8.5 years vs. median not reached; $p=0.014$; Fig. 3 ), as well as a decrease in 5 -year distant control (46.9 vs. $91 \%$; median 4.2 years vs. median not reached; $p=0.017$; Fig. 4 ). 


\section{Discussion}

PDTC is a rare and heterogenous type of thyroid cancer. Approximately 20 years have passed between its first description in 1983 [12], and 2004, when it was incorporated in the WHO Classification of Thyroid Tumors [6]. Nevertheless, the diagnostic criteria still generate controversy and the WHO classification has been difficult to implement in routine clinical practice [7]. In 2006, the Turin Consensus Group proposed a more practical diagnostic algorithm of PDTC [11]. The prognostic role of serum Tg levels obtained after initial treatment of DTC is well established [13]. On the other hand, until the study by Ibrahimpasic et al. [9], the prognostic value of Tg levels in PDTC was unclear. These authors specifically studied the role of $\mathrm{Tg}$ as a possible prognostic factor in 31 PDTC cases, showing that patients with detectable Tg levels after surgery and RAI had an increased margin for positive disease and higher/earlier recurrence rates. The PDTC cases were classified based on the Memorial Sloan Kettering Cancer Center (MSKCC) criteria, which differs from the Turin proposal as it only requires necrosis or a high mitotic index, regardless of growth pattern and nuclear features [14]. Despite these differences, Gnemmi et al. [15] showed that these 2 sets of criteria similarly predict an intermediate prognosis for PDTC cases (between DTC and anaplastic thyroid carcinoma).

Our study supports and expands the prognostic role of serum Tg obtained after initial treatment (surgery + RAI) in PDTC. We confirm that Tg levels can predict morbidity [9] and, in addition, we show for the first time that mortality can also be predicted by serum Tg in these patients. In fact, in our cohort, the group with detectable Tg after surgery and RAI had a significantly worse 5-year DSS than patients with undetectable Tg (65 vs. $100 \%$, median 11.2 years vs. median not reached; $p=0.009$ ). In the former group, 2 deaths were observed in the first year of follow-up while no death occurred in the first 8.7 years in the latter group. A more aggressive disease course compared to that described by Ibrahimpasic et al. [9] (a 5-year DSS in the detectable Tg group of 65 vs. $95.8 \%$ in the undetectable Tg group), associated with a larger sample size, and longer follow-up time, may explain why specific survival was only significantly correlated with serum Tg levels in our study.

We also found a reduced 5-year RFS in the group with detectable $\operatorname{Tg}$ ( 32 vs. $84 \%$; median 1.7 years vs. median not reached; $p=0.010$ ). Both locoregional and distant control were also significantly worse with detectable $\mathrm{Tg}$ (locoregional RFS of 55.6 vs. $90.9 \%$; median 8.5 years vs. median

Increased Mortality and Recurrence in PDTC with Detectable Tg Levels not reached, $p=0.014$; distant RFS of 46.9 vs. $91 \%$; median 4.2 years vs. median not reached, $p=0.017$ ). The group with detectable $\mathrm{Tg}$ had $70 \%$ of its recurrences ( 7 cases) in the first year of follow-up. On the other hand, the group with undetectable Tg levels had 4 recurrences, 2 (50\%) in first year. The patients with detectable Tg levels also had a significant increase in positive surgical margins after surgery ( 46.6 vs. $21.7 \%, p=0.004$ ); for the first time, we have also shown a significant increase in positive nodal disease in the neck in this group ( 73.3 vs. $21.8 \%, p=0.005$ ). Other pathological characteristics such as a pT4 status (61.1 vs. $37.9 \%, p=0.083)$ and extrathyroidal extension (80.0 vs. $52.2 \%, p=0.082$ ) only showed a trend towards higher prevalence in the group with detectable Tg levels.

Our study has some limitations. The retrospective study design associated with a clinical heterogeneous disease and a small sample size (38 patients) can limit the applicability of the study conclusions. Nevertheless, despite being a small sample, it comes from a single center with a large series of PDTC cases (101 patients, unpublished data) and is, so far, the largest series evaluating the role of Tg in PDTC patients; it also had the longest followup time. Furthermore, the results broadly agree with a recent paper published by Ibrahimpasic et al. [9].

In summary, we conclude that Tg levels in PDTC M0 patients after treatment with surgery and adjuvant RAI have a prognostic value similar to patients with DTC. Those with detectable Tg levels have more aggressive disease, and higher rates of persistent microscopic disease after surgery with higher rates of nodal and metastatic recurrence lead to a reduced OS. Therefore, serum Tglevels may be a useful marker for early recurrence and death in PDTC. However, although nonsuppressed Tg levels seem to predict a poorer outcome, suppressed Tg levels may fail to reveal early recurrence. We emphasize the need for larger multi-institutional studies to confirm the role of detectable Tg levels in the morbidity and mortality of PDTC patients.

\section{Acknowledgements}

iNOVA4Health UID/Multi/04462/2013, a program financially supported by Fundação para a Ciência e Tecnologia Ministério da Educação e Ciência, through national funds and co-funded by FEDER under the PT2020 Partnership Agreement is acknowledged.

\section{Disclosure Statement}

The authors declare they have no conflict of interest. 


\section{References}

1 Faggin JA, Wells SA: Biologic and clinical perspectives on thyroid cancer. N Engl J Med 2016;375:1054-1067.

2 Sanders EM Jr, LiVolsi VA, Brierley J, Shin J, Randolph GW: An evidence-based review of poorly differentiated thyroid cancer. World J Surg 2007;31:934-945.

3 Ibrahimpasic T, Ghossein R, Carlson DL, Nixon I, Palmer FL, Shaha AR, et al: Outcomes in patients with poorly differentiated thyroid carcinoma. J Clin Endocrinol Metab 2014;99:1245-1252.

4 Volante M, Landolfi S, Chiusa L, Palestini N, Motta M, Codegone A, et al: Poorly differentiated carcinomas of the thyroid with trabecular, insular, and solid patterns: a clinicopathologic study of 183 patients. Cancer 2004;100: 950-957.

5 Landa I, Ibrahimpasic T, Boucai L, Sinha R, Knauf JA, Shah RH, et al: Genomic and transcriptomic hallmarks of poorly differentiated and anaplastic thyroid cancers J Clin Invest 2016;126:1052-1066.

6 DeLellis RA, Lloyd RV, Heitz PU, Eng C (eds): World Health Organization Classification of Tumours: Pathology and Genetics of Tu- mours of Endocrine Organs. Lyon, IARC, 2004.

7 Volante M, Bussolati G, Papotti M: The story of poorly differentiated thyroid carcinoma: from Langhans' description to the Turin proposal via Juan Rosai. Semin Diagn Pathol 2016;33:277-283.

8 Killeen RM, Barnes L, Watson CG, Marsh WL, Chase DW, Schuller DE: Poorly differentiated ("insular") thyroid carcinoma. Report of two cases and review of the literature. Arch Otolaryngol Head Neck Surg 1990;116:10821086.

9 Ibrahimpasic T, Ghossein R, Carlson DL, Nixon IJ, Palmer FL, Patel SG, et al: Undetectable thyroglobulin levels in poorly differentiated thyroid carcinoma patients free of macroscopic disease after initial treatment: are they useful? Ann Surg Oncol 2015;22:41934197.

10 Edge SB, Byrd DR, Compton CC, Fritz AG, Greene FL, Trotti A (eds): AJCC Cancer Staging Manual, ed 7. New York, Springer, 2010.

11 Volante M, Collini P, Nikiforov YE, Sakamoto A, Kakudo K, Katoh R, et al: Poorly differentiated thyroid carcinoma: the Turin pro- posal for the use of uniform diagnostic criteria and an algorithmic diagnostic approach. Am J Surg Pathol 2007;31:1256-1264.

12 Sakamoto A, Kasai N, Sugano H: Poorly differentiated carcinoma of the thyroid. A clinicopathologic entity for a high-risk group of papillary and follicular carcinomas. Cancer. 1983;52:1849-1855.

13 Cooper DS, Doherty GM, Haugen BR, Kloos RT, Lee SL, Mandel SJ, et al: Revised American Thyroid Association management guidelines for patients with thyroid nodules and differentiated thyroid cancer. Thyroid 2009; 19:1167-1214

14 Hiltzik D, Carlson D, Tuttle RM, Chuai S, Ishill N, Shaha A, et al: Poorly differentiated thyroid carcinomas defined on the basis of mitosis and necrosis: a clinicopathologic study of 58 patients. Cancer 2006;106:1286-1295.

15 Gnemmi V, Renaud F, Do Cao C, Salleron J, Lion G, Wemeau JL, et al: Poorly differentiated thyroid carcinomas: application of the Turin proposal provides prognostic results similar to those from the assessment of highgrade features. Histopathology 2014;64:263273 